\title{
WATER SUPPLY REMOTE MONITORING SYSTEM: A CASE STUDY
}

Keywords: Remote Monitoring, water supply, GSM, Programmable Logic Controller

Abstract: In recent years, there has been enormous research interest in natural resources monitoring. So, there is a need to develop easily accessible, cheap and reliable information systems for monitoring and early warning, which could be used in most natural resources. This paper presents an ongoing information system development that aims the monitoring and supervising of some parameters on water supply, such as quality and quantity. To implement our system we use several technologies in order to monitoring about 100 water tanks in an area of $\pm 1000 \mathrm{Km}^{2}$. In these technologies is included GSM communication, web infrastructure and sensing equipment that allows the access of information from any place of the world. In paper sections the main blocks of the system architecture are described in detail.

\section{INTRODUCTION}

Water quality is an important indicator of our quality of life. Is important to guarantee that water never lacks or loses quality. Monitoring and controlling levels of some contaminants in drinking water are important steps towards preventing human health problems. Even though drinking water, including bottled water, may reasonably be expected to contain at least small amounts of some contaminants this does not necessarily indicate that the water poses a health. However, high levels of some contaminants can improve the risk of infection, particularly in people who are more vulnerable to contaminants in drinking water than the general population, such as immunocompromised people with cancer undergoing chemotherapy, people who have undergone organ transplants and people with immune system disorders. (University of Notre Dame, 2003)

Nowadays water quality is a real concern both population and water suppliers. They are concerned with questions like: How good is the water I drink today? Is the water quality becoming better or worse? What is making the water quality better or worse, and how can that improve? What does a water treatment plant need to do to make the incoming water drinkable?
Even though most cities usually have goodquality water, there are several concerns:

- Concentrations of metals and acids in the water are high risk.

- High nutrient levels in water can cause algae blooms and lower the amount of oxygen in water. This causes the water (even when treated) to taste strange.

- High amounts of sediment (sand, gravel, or ash from a burned area) clog the gills of fish and the filters of drinking water treatment plants.

Water supply to different populations in sufficient quantities, with the best quality and the most reasonable costs, must be one of the great concerns of the people responsible for this area. The major difficulties are the situation of the water tanks, access difficulty, electric power supply and difficulty of communication between some stations and those responsible for the water supply. Some remote stations (one or more water tanks) can be in isolated places, with difficult access, and often do not have power supply. On the other hand, the distance between the station and the responsible entity can be huge. So that the responsible entity has reliable and up-to-date information about each water tank, as well as the amount and quality of the water, it is necessary to implement a system that answers to these questions. 
The work presented in this paper was guided by the design and implementation of an information system to monitor some parameters related with water supply.

This work has being developed under an academic research project driven by undergraduate students in collaboration with water supplier.

In order to model the information system developed, an object-oriented approach was followed, namely the UML - Unified Modelling Language (Booch et al., 1999).

This paper is structured as follows. The following section describes the problem. An overview of the main requirements for the system developed is presented in section three, and our prototype is presented in section four Finally, we will summarise our results and make a brief reference to some topics for future work.

\section{THE PROBLEM}

In some cases water quality monitoring is done by an individual that visits each water tank and takes samples. Often these water tanks are in places with difficult access being, making the procedure difficult and expensive and results unreliable.

In this case we have just about one hundred water tanks dispersed in an area with approximately $1000 \mathrm{Km} 2$. Obtain samples of all water tanks manually, analyse each sample and make changes in water quality control, if needed, is a slowly and expensive task that not guarantees reliable results. Sampling results obtained with a big delay doesn't let doing corrections in time. One way to minimize this problem is through an information system that allows remote monitoring of some water properties.

Real time remote sensing of the water parameters provides information that enables effective monitoring and control of water quality at low cost. All this information is periodically transmitted to the head office and stored in a database. The system may be equipped with decision support algorithms and applied to identifying and controlling changes in each water tank's properties that are significant in improving water quality. The consultation and administration of the data will be carried through the Internet.

The information system will allow real time monitoring and notice about some atypical situations. With real time information, it will be possible to prevent the supply imperfections of impure water to the populations, as well as to report on and statistically analyse the water levels.

We split this project in two different stages. On stage 1 we aim to remotely monitoring the water and
$\mathrm{pH}$ levels on each tank. At second stage the main goal is enlarge the range of controlling fields, such as chlorine.

Having a continuously monitoring of the water properties of each water tanks it is possible to act much more quickly and with better results.

\section{OVERVIEW OF SYSTEM REQUIREMENTS}

\subsection{Main System Requirements}

The developed system should deliver the required functionality and performance to the user and should be maintainable, dependable and usable. In the remote monitoring environment, it is crucial to provide fast, reliable and on-time responses when dealing with unexpected events. These are the most important high-level and general requirements to be fulfilled by the system.

System requirements are usually divided into two classes - functional requirements and nonfunctional requirements. The first describe what the system should do and are perceptible to the user, while the second describe constraints on how the functional requirements are implemented, and are not necessarily perceptible by the user (Sommerville and Sawyer, 1997).

\subsection{Functional Requirements}

Remote configuration. Through this requirement it is possible to configure some parameters of the stations remotely, namely the frequency sampling of each sensor.

Event Notification. This feature is considered a very important requirement, because it allows notification in real-time if disruptive events occur in one station, e.g. if the water level of one tank gets down the set-point, the system sends an alert to the person in charge of the maintenance.

Update Information. The system must allow inquiry into stations in order to attain current data. This will allow information of the status of any station and its sensors in real-time.

Monitoring stations status. One efficient strategy to reduce the risk of problems in water supply is by better controlling aspects such as the level and quality of the water. Monitoring the stations brings two major benefits, namely real-time analysis of these parameters and using the data to produce statistical reports. 


\subsection{Non-Functional requirements}

Performance. The system response time depends on how sophisticated the sensors are. If the sensors are rough (level sensors), the system will be cheaper but not so accurate when using sophisticated sensors (ultra-sonic).

Flexibility. The system must be flexible in order to allow the user to insert, remove or edit elements, such as new stations, more sensors or adding mobile phone numbers to deliver alerts.

Usability. A friendly interface, flexible, with strong graphical capabilities and succinct and clear messages can raise the system efficiency.

Power supply. In order to solve the problem of remote stations located in isolated places, with difficult access, and without power supply, all these stations need to be equipped with a solar panel and a battery.

\subsection{Use Case diagram}

One of the first steps considered in the modelling was to describe the system as a number of use cases that are performed by a set of actors.

A Use Case diagram presents a set of use cases, actors and their relations. Their common applications are usually divided into two - system context modelling and system requirements modelling. The former gives emphasis to the identification of the boundary system, their actors and the meaning of their functions, while the second consist of the identification of what the system should do, no matter how. Figure 1 and 2 illustrate some of the Use Case diagrams considered for the system.

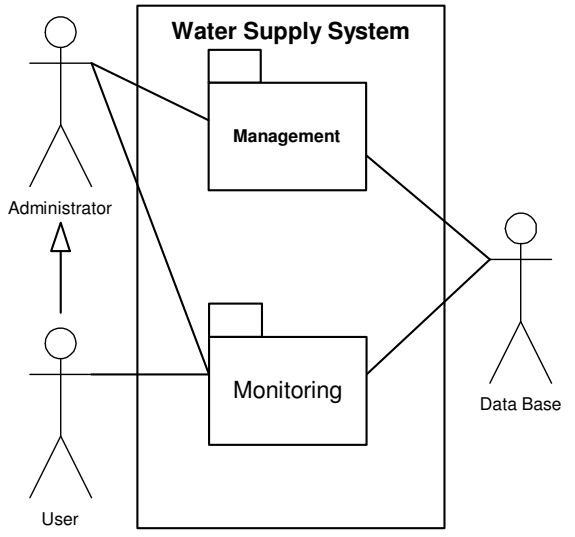

Figure 1: Use Case Package diagram for the proposal system.

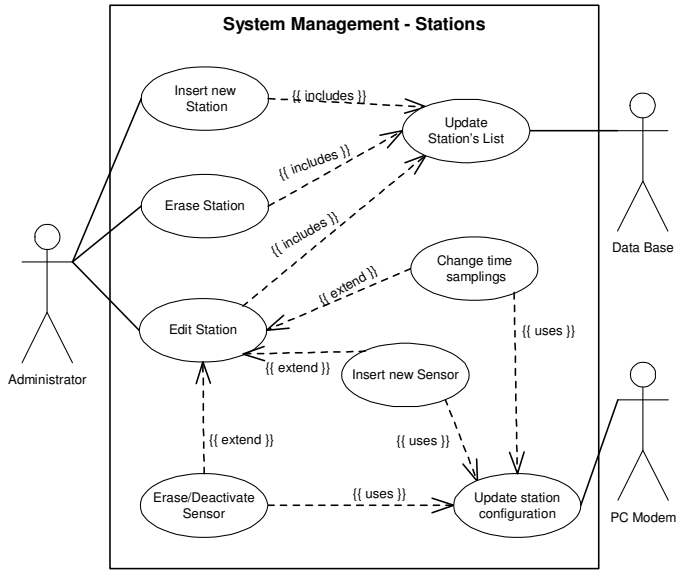

Figure 2: Use Case diagram for Management Package.

\section{PROTOTYPE}

\subsection{Architecture}

The proposed system uses commercial hardware to allow the acquisition of data from several sensors (water level, $\mathrm{pH}$ ) placed inside/near to the watertank (properly protected against dust and other bad effects of the environment). After the data acquisition, with a defined sampling period, this data is stored and sent by a remote station to the head office station, placed in the central services office. The responsible for the water resources maintenance is advised every time that a disruptive event (ex. water fault in a water-tank). There are several ways to get information about the tanks status. The first is by a messages sent to a mobile phone, through Short Message Service (SMS) using Global System Mobile Communications (GSM) technology, every time that a disruptive event occurs. The user can also get data in real-time by polling the desire remote stations. Finally is also possible to consult data stored in the database making possible the analyses and reports of the historical acquired data.

All this features can be accessed at any time from a remote place, even from outside Portugal by the World Wide Web site, through an Internet link. To implement this feature we used PHP technology. With this access the user can consult the data stored in the host PC, located in the central monitoring office and define the set-points for the controlled variables. The architecture of the implemented system is showed in Fig. 3. 


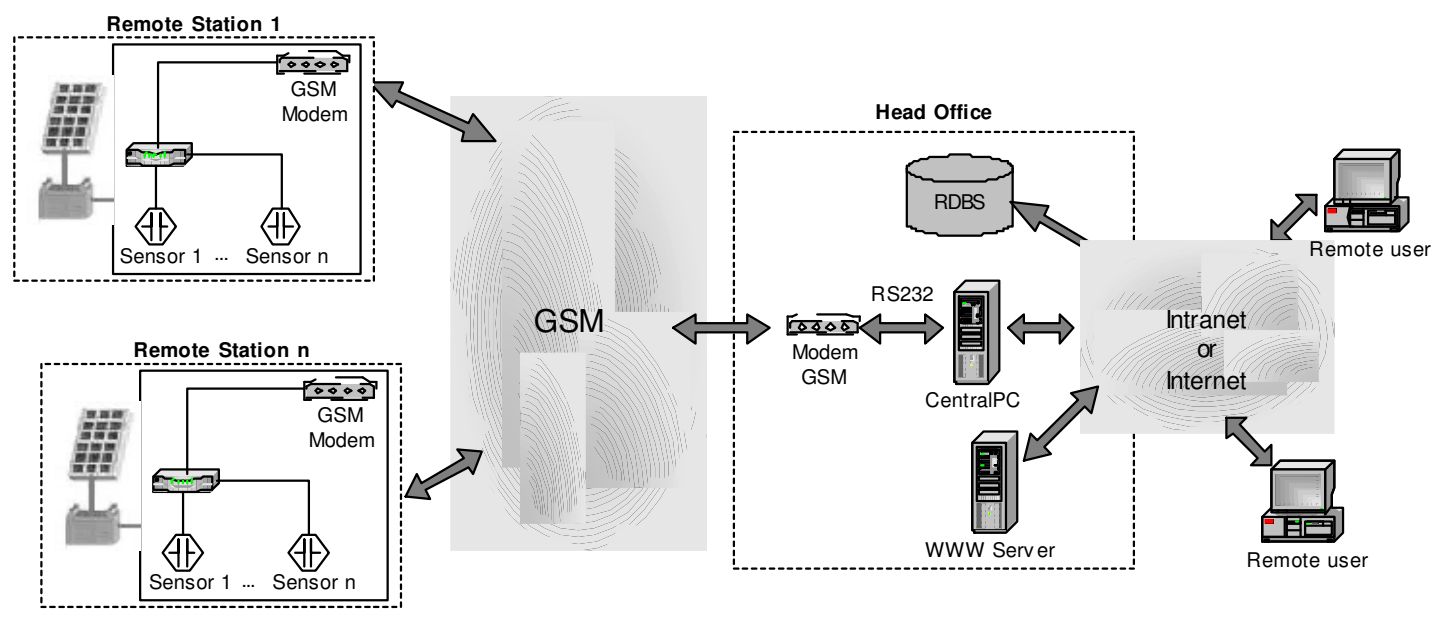

Figure 3: Architecture of the proposal system.

\subsection{System Implementation}

To implement the proposed system, commercial hardware was used and software was developed to reach the desired goals. In the next part of this paper we will summary expose the main blocks.

\section{Hardware}

To acquire data we use a Programmable Controller (FPO-T32CP, 2003) that acts as an interface between the sensors, physic world, and a GSM modem. The used programmable controller will allow, in a future stage of the system development, digital control over actuators like valves or others. Some features of the used controller are:

- 32 I/O points (16 Input and 16 Output) with expansion until $128 \mathrm{I} / \mathrm{O}$ points;

- Program capacity of $10 \mathrm{~K}$ steps;

- Operation speed (central value/step) of $0.9 \mathrm{~ms}$;

- Programme memory uses EEPROM or RAM;

- 100 subroutines;

- Several special functions;

- self-diagnosis functions;

- Password setting;

- Run time editing;

- Built-in functions for interrupt processing, high speed counting and pulse output;

- 24 VDC operating voltage;

- RS232 serial port for connection to intelligent devices or modems for telemetry applications.
In this acquisition stage commercial level sensors had been used to read the water level values inside the water tank.

To allow the communication between remote places and the central system two types of GSM modem were used. One from Matsuhita (Fastrack, 2004) and one from siemens (M20, 1999) whose features are detailed in the referenced documents and web sites.

The implemented remote station prototype is showed in the following photo:

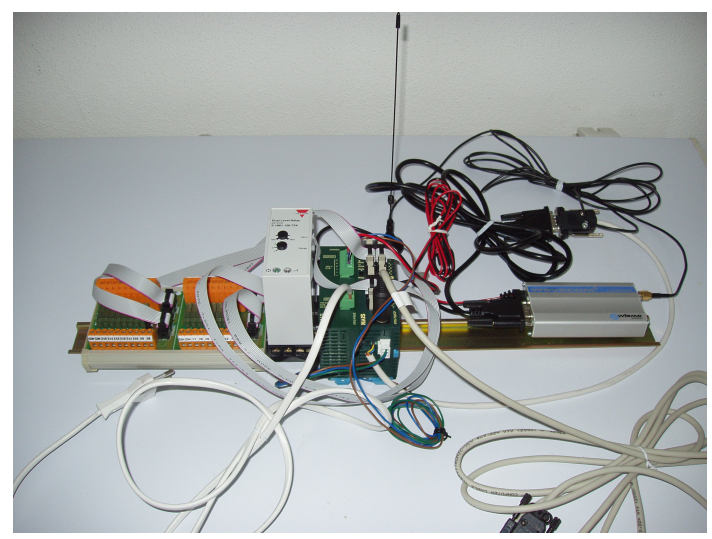

Figure 4: Photo of the implemented remote station prototype.

\section{Software}

The software component of the system uses several different languages/technologies: mySQL (MySQL, 2004), PHP (PHP, 2004), HTML, Control FPWIN Pro (Control FPWIN Pro, 2003) Its usage is: 
- MySQL is used as support of the database, where the data read by the PLC is stored, but also to allow queries to the database, for its manipulation. The data manipulation is basically the actions of average calculation, maximum value calculation, minimum value detection, data sort, etc.

- PHP is used to allow the connection with the database. The connection is done using an ODBC redirected to the database.

- HTML was used to build a WEB page. This page allows in "real time" the graphical presentation of the data read by the logger. HTML also uses PHP to accede to the database, using SQL queries embedded in PHP.

- FPWIN Pro is used in the remote acquisition station to allow the acquisition of data from sensors and transmission of the sampled data to the central system. This tool is Matsushita programming software. It allows 5 different languages (instruction list. Ladder diagram, structured text, function block diagram and sequential function chart).

\section{CONCLUSIONS AND FURTHER WORK}

The results of the stage one of the described work are uncourageous.

Tests have been made regarding the remote station based on the defined message protocol. With also test alarm conditions scenarios, namely miss of water and high $\mathrm{pH}$ level. The results have been excellent.

On the head office block of the system the data storage, the SMS communication and the reports analyses are also implemented.

Further work will involve the refinement of the remote stations, namely stage two, i.e. adding more sensing and actuation equipment. We aim to test the system under full charge (critical conditions).

We plan to have the full developed system in July 2004.

\section{REFERENCES}

Booch, G., Rumbaugh, J. and Jacobson, I. (1999). The Unified Modeling Language User Guide, Addison Wesley.

Control FPWIN Pro, Programmable Controller FPO catalogue, Matsuhita Electric Works, 2003. [online] Available: www.matsuhita.es, catalogue page 19
Fastrack M1203A-ON GSM/GPRS Modem, www.wavecom.com [website consulted in 26February-2004].

FPO-T32CP, Programmable Controller FPO datasheet, Matsuhita Electric Works, 2003. [Online] Available: www.matsuhita.es, catalogue page 17.

M20 Cellular Engine Siemens terminal, Siemens Information and Communication products, Version 5, 1999.

MySql Ab. [online]. Available: http://www.mysql.com/ PHP. [online]. Available: http://www.php.net/

Sommerville, I. and Sawyer, P. (1997). Requirements Engineering: A Good Practice Guide, John Wiley \& Sons. Chichester.

University of Notre Dame (2003), Annual Drinking Water Quality Report, Consumer Confidence Report 2003, available: www.nd.edu/ riskman/manuals/documents/ waterqualityrpt2002_000.doc 